\title{
e-Phaïstos
}

e-Phaïstos

Revue d'histoire des techniques / Journal of the history

of technology

IV-2 | 2015

Patrimoines de l'eau

\section{Valorisation et interprétation du patrimoine hydroélectrique dans le paysage contemporain en Italie}

Valorization and interpretation of the hydroelectric heritage in the contemporary landscape in Italy

\section{Erika Bossum}

\section{OpenEdition}

Journals

Édition électronique

URL : http://journals.openedition.org/ephaistos/765

DOI : 10.4000/ephaistos.765

ISSN : 2552-0741

Éditeur

IHMC - Institut d'histoire moderne et contemporaine (UMR 8066)

Édition imprimée

Date de publication : 1 octobre 2015

Pagination : 60-70

ISSN : 2262-7340

Référence électronique

Erika Bossum, « Valorisation et interprétation du patrimoine hydroélectrique dans le paysage contemporain en Italie », e-Phaïstos [En ligne], IV-2 | 2015, mis en ligne le 29 novembre 2016, consulté le 10 décembre 2020. URL : http://journals.openedition.org/ephaistos/765 ; DOI : https://doi.org/ 10.4000/ephaistos.765 


\section{Valorisation et interprétation du patrimoine hydroélectrique dans le paysage contemporain en Italie.}

\begin{abstract}
«Il tempo ha lavorato a favore dei nuovi paesaggi ; quelli originari sono stati dimenticati, le nuove generazioni non li hanno mai visti ${ }^{1}$
\end{abstract}

\section{Introduction}

En 1998, Rosario Pavia décrit ainsi les paysages électriques, des zones historiquement utilisées pour la production d'énergie hydroélectrique, devenues depuis longtemps parties intégrantes des paysages italiens. La participation des ouvrages hydroélectriques dans la construction du paysage a été consolidée depuis des décennies et leur impact sur l'environnement a été intériorisé, absorbé2.

Aujourd'hui, de nouvelles valeurs sont affectées à ces lieux. Ce sont des valeurs qui appartiennent aux secteurs des disciplines de l'histoire et de la connaissance technologique, économique, sociale, environnementale. En ce qui concerne le domaine des nouvelles technologies, l'eau, qui est considérée depuis toujours comme un bien économique3, est de plus en plus, à l'heure actuelle, une ressource stratégique. Dans une optique de développement durable, l'exploitation de l'eau comme énergie dans les pays en développement est utilisée pour l'autosuffisance dans les zones où il y a inefficacité énergétique4. Cela se passe aussi dans les vieux pays

\author{
Erika Bossum \\ Master Erasmus Mundus TPTI, \\ promotion 1 « Historiens sans \\ frontières » \\ Doctorante, Università degli Studi di \\ Verona
}

industrialisés, où il $\mathrm{y}$ a une tendance à l'implantation de nouvelles centrales 5 ou à la restauration de centrales désaffectées ${ }^{6}$, en exploitant les anciens réseaux de canalisation. À cet égard, le Mini Hydro ${ }^{7}$ est une tendance répandue au niveau mondial.

En Italie, ce regain d'intérêt dans les zones historiquement consacrées à la production d'énergie est soumis à l'évolution du débat autour de la notion de paysage ${ }^{8}$. Depuis le début du débat sur le paysage $^{9}$ en Italie, ainsi que dans le reste de l'Europe, qui s'est fait avec des dynamiques différentes, les paysages eux-mêmes ont subi de profonds changements. En général, dans les villes italiennes, il $\mathrm{y} a$ eu une première phase d'urbanisation en dehors des murs de la ville, qui a dégénéré en urban sprawl dans la période postindustrielle et qui se caractérise par l'utilisation de terrains dédiés à l'édification d'une qualité douteuse ${ }^{10}$. Ce processus a suscité des réactions importantes, donnant naissance à une culture du paysage. Dans ce contexte, on garde espoir pour l'avenir des lieux qui n'ont pas encore été cimentés. On assiste à la redécouverte et à la relecture des lieux de production d'énergie, dont l'isolement a permis de sauver la nature de l'intervention de 
l'urbanisation, même s'il s'agit d'un contexte fortement caractérisé par la présence de l'industrie hydroélectrique. Ce sont les endroits où l'eau a été détournée au détriment des voies navigables historiques. Dans ces lieux, l'eau coule encore en surface, grâce à leur localisation périphérique et inaccessible, dans des canaux qui ont été sauvegardés à la " mise à mort de l'eau » ${ }^{11}$, imposée dans les noyaux urbains historiques. Il s'agit d'une "seconda natura »12, antropizzata et contemporaine, qui peut accueillir une nouvelle façon d'habiter, soutenable et culturellement stimulante.

Si dans un premier moment, le débat sur le paysage a été vivement animé par la prise de position des chercheurs, des historiens et géographes qui ont condamné le rôle de l'industrie dans la destruction de l'équipement d'un paysage rural typiquement italien, on est arrivé à l'idée que les régions d'ancienne industrialisation liées à l'exploitation des eaux offrent également un territoire de protection pour les connaissances en science et technologie ${ }^{13}$.

En outre, ces sites présentent un intérêt pour le développement de la société moderne, qui y reconnaît des nouveaux lieux de loisirs ${ }^{14}$, où le mélange de nature artificielle et des éléments architecturaux deviennent des conteneurs pour le développement culturel, avec des effets intéressants sur l'économie locale. Les biens du patrimoine industriel pour la production hydroélectrique deviennent partie intégrante des parcs urbains: des pistes cyclables sont créées sur les bords des canaux, les centrales hydroélectriques deviennent des stations-services et parfois il est possible de visiter l'intérieur des bâtiments ou même d'y assister à des spectacles culturels. Les biens du paysage hydraulique, même s'ils sont présents sur le territoire depuis des décennies, deviennent accessibles lorsque les entités qui gèrent les systèmes interagissent avec les institutions locales. De cette manière, les institutions ont la capacité de démarrer un processus qui aboutira à la réintégration de ces lieux dans les dynamiques du développement urbain. La diffusion des connaissances, la mise en place des espaces de musée, la transmission des valeurs historiques et culturelles, l'interprétation du territoire, ne sont que quelques-uns des objectifs atteints dans le processus de patrimonialisation.

Dans la constellation d'expériences de valorisation du patrimoine hydroélectrique, il a été décidé de présenter pour exemple les cas qui représentent une façon d'interpréter et de transmettre les connaissances et la culture liées aux biens en question. Le choix de ces expériences est motivé par le fait qu'ils sont en fait potentiellement réalisables à Vérone, cas qui sera examinée dans le deuxième chapitre ; Vérone dispose d'infrastructures, systèmes et réseaux historiques, comparables à ceux examinés. Les types de mesures présentées ci-dessous ont été mis en œuvre dans les nord de l'Italie, et plus précisément dans les régions de la Vénétie, Trentin-Haut-Adige et la Lombardie, avec des caractéristiques géographiques et géomorphologiques similaires à ceux de Vérone.

\section{Exemples de mise en valeur en Lombardie, Trentin-Haut-Adige et Vénétie}

\section{Itinéraires informatiques interactifs}

Les ressources informatiques jouent un rôle stratégique pour la mise en évidence des éléments du paysage. Quand ils sont mis en relation les uns avec les autres, ces objets deviennent des composantes de systèmes territoriaux complexes.

Précurseur fut le projet The Way of EnergyLombardy ${ }^{15}$, sous le patronage de la Région Lombardie, et créé par Arte.Media.Scienza (Ar.Me.S.) ${ }^{16}$ et l'Université de Milan Bicocca ; cette plateforme focalise les réseaux énergétiques et ses monuments historiques. C'est un instrument de connaissance mis en ligne qui possède une double fonction: fournir un support à l'enseignement et proposer des itinéraires adressés aux usagers qui 
sont intéressés par le patrimoine hydroélectrique et industriel en général. Le site web offre également la possibilité de connaître les cultures locales, y compris l'architecture, la gastronomie et le folklore, des informations sur les politiques environnementales investies dans le territoire ${ }^{17}$ conçu dans le cadre d'un système vivant grâce à la richesse de preuves historiques.

\section{Parcs et écomusées sur le patrimoine hydroélectrique}

A niveau national, la plateforme «parks.it» offre une base de données entre le plus remarquables concernant la connaissance de différentes réalités territoriales et leur promotion culturelle. Le parc Adda Nord ${ }^{18}$ est un cas exemplaire. Il a été créé en 1983 par la Région Lombardie et est géré par un consortium de 32 municipalités situées dans les provinces de Bergame, Lecco et Milan. L'office du parc est responsable des mesures les plus traditionnelles de protection des aspects naturels et environnementaux. Il s'occupe également d'un certain nombre d'initiatives pour la protection, la conservation et la valorisation de la présence importante du patrimoine industriel. En outre, il gère les itinéraires touristiques le long de l'Adda et l'équipement d'un certain nombre d'endroits pour pratiquer des sports de différents types. Il y a cinq centres de visite du parc offrant des points d'information, des plaques didactiques, des bibliothèques, une ferme pédagogique et qui organisent des conférences et des expositions.

Le parc Adda Nord est un cas réussi si l'on considère la façon avec laquelle on a eu la capacité d'interpréter et de comprendre la complexité de la sédimentation historique et culturelle présente dans le parc. Les aspects archéologiques et monumentaux, par exemple, sont particulièrement mis en valeur. Il s'agit des œuvres de génie hydraulique du début du XXe siècle, sur des modèles de barrage projetés ici par Léonard de
Vinci, qui a vécu dans ces lieux ${ }^{19}$.

Le parc Adda Nord comprend huit centrales hydroélectriques, construites entre Brivio (Lecco) et Rivolta d'Adda (Cremona) à partir de 1895 et ce jusqu'en 1928. Aujourd'hui, la présence de ces témoignages combinée à une indéniable qualité du contexte naturel fluvial, fait de cet endroit un excellent exemple de la sauvegarde du paysage et de l'environnement.

Le fleuve Adda, à courant torrentiel, a empêché l'industrialisation jusqu'à ce que la Société Edison réalise les premières œuvres de canalisation pour la production d'énergie hydroélectrique. Les centrales hydroélectriques Bertini (1895) ${ }^{20}$ Esterle (1906) ${ }^{21}$ et Semenza $(1917)^{22}$, qui fonctionnent encore, ont permis le développement d'activités industrielles, telles que l'usine Velvis de Vaprio d'Adda (Milan). Le parc est confiné au nord par le pont ferroviaire de Paderno (1887-1889) ${ }^{23}$ et il fait partie des itinéraires culturels qui incluent le village de Crespi d'Adda (Bergame)24, site classé au patrimoine mondial de l'UNESCO depuis 1995.

Le patrimoine de l'eau pour la production d'énergie est un dénominateur commun du musée en plein air d'archéologie industrielle de Schio (Vicenza), dans la région de la Vénétie. Ces objets d'exposition constituent des monuments industriels largement diffusés sur le territoire, y compris les centrales hydroélectriques de Val Leogra, Molino di Poleo, Savardo. Le musée comprend également plusieurs itinéraires pour visiter les industries de filature de laine, le nouveau quartier ouvrier de Schio, les canaux d'irrigation, les scieries, les moulins, les mines de kaolin, l'usine Saccardo, l'ancien domaine d'Alessandro Rossi en Santorso, le village et la brasserie industrielle Summano de Piovene Rocchette.

Le système d'écomusée a été amélioré en 2011, à travers le projet Musées Alto Vicentino, qui permet la mise en réseau de plusieurs musées, réunis sous un même nom, pour donner de la vigueur aux activités culturelles d'un territoire qui est raconté à travers son patrimoine ${ }^{25}$. 


\section{Routes dans les espaces urbains, collinaires et montagne}

En 1998, Rosario Pavia dénonçait comment les guides de montagne excluent les éléments artificiels des routes, dans l'intention de sélectionner l'environnement, le purifier des traces infrastructurelles ${ }^{26}$. Comme déjà mentionné, au cours des dernières années, la sensibilité s'est modifiée et aujourd'hui l'attention est également accordée au patrimoine hydroélectrique de la colline et de la montagne. À titre d'exemples il suffit de citer: les itinéraires du parc des cascades des Marmore ${ }^{27}$, dans la province de Terni, en Ombrie; les chemins qui conduisent aux centrales-musées de Gais, Kurtatsch ${ }^{28}$, ou Fortezza ${ }^{29}$, dans la province de Bolzano; le Musée de la Technologie Tecneum de Brixen ${ }^{30}$, en Tyrol du Sud, pour être en mesure de dire que non seulement les routes urbaines, mais aussi de nouvelles initiatives dans les collines et dans les zones de montagne, sont maintenant très répandues.

D'une manière analogue, plusieurs initiatives peuvent animer les espaces urbains de la production hydroélectrique. Souvent, les mêmes sociétés historiques qui gèrent les centrales hydroélectriques sont les promotrices des activités culturelles qui s'y déroulent. Dans la ville de Milan, par exemple, opère la Fondazione $A E M^{31}$. La fondation a été créée par la même entreprise que celle qui s'occupe de la gestion énergétique municipale de Milan, maintenant appelé A2A ${ }^{32}$. En collaboration avec le Politecnico di Milano, la Fondazione AEM organise des visites dans les quartiers historiques de la ville, pour connaître les anciens lieux de production d'électricité.

\section{Itinéraires à travers les images photographiques}

La photographie est un outil de communication et de documentation efficace qui permet un nombre infini d'itinéraires thématiques dans l'architecture, l'aménagement paysager, la sociologie et la technologie. Le photographe crée des documents exclusifs, dans les endroits les plus inaccessibles. Il s'agit d'instantanés des centrales dans une perspective très rapprochée ou panoramique permettant de considérer l'étendue et la dimension des systèmes énergétiques.

À cet égard, le projet de catalogage concernant les ouvrages hydroélectriques répartis dans la région du Trentin est exemplaire. Le recensement photographique a été l'objet de l'exposition «Acquaenergia. Storia e catalogazione delle centrali idroelettriche in Trentino $\gg 33$, qui s'est tenue en 2008. Le travail effectué a permis de documenter l'état actuel des centrales hydroélectriques de la région.

Le patrimoine photographique est un élément important dont il faut tenir compte dans l'analyse de l'évolution du paysage. La prise de photo lors de la construction d'œuvres d'art, ou suite aux dévastations de guerre, même sujette à l'interprétation particulière du photographe contemporain compte de manière importante pour la connaissance de certaines sociétés.

Photographies, films, documents et matériel iconographique ont été l'objet de l'exposition «I luoghi dell'acqua. Architetture e paesaggi delle centrali elettriche in Valtellina » qui s'est tenue à Sondrio, en Lombardie, en février $2013^{34}$.

L'exposition «I luoghi dell'acqua » a proposé les images de plusieurs dizaines d'ouvrages d'art pour la production d'énergie, réalisés au début du $\mathrm{XX}^{\mathrm{e}}$ siècle et toujours en place. Le but de l'exposition était de montrer comment la modernité, représentée par l'architecture des centrales, a introduit un nouveau langage stylistique dans le paysage traditionnel de la Valtellina. Centrales, barrages, bassins de retenue, conduites forcées, canaux, villages ouvriers, constituent le véritable paysage artificiel qui reflète le renouveau architectural et urbain dans la province de Sondrio et les vallées voisines. Il s'agit d'œuvres signées par 
de célèbres architectes italiens, Gio Ponti et Giovanni Muzio, ainsi que des designers moins connus qui ont travaillé dans les bureaux de Edison Falck technique et AEM.

\section{Manifestations culturelles dans les centrales encore en activité}

Il s'agit de cas dans lesquels les centrales, tout en continuant à produire de l'énergie, proposent des activités culturelles d'un type expérimental et créatif.

Parmi ces activités, Centrale Fies est un projet d'intérêt international. La centrale de Fies est située à proximité de Dro, une localité dans la province de Trento, région Trentin. Construite au début du XX ${ }^{\mathrm{e}}$ siècle le long de la rivière Sarca, la centrale est du type centrale-lac et elle est encore en fonctionnement, ce qui lui donne une double fonction. En effet, en plus de l'hydroélectricité, la centrale de Fies est également un centre pour la production d'œuvres d'art contemporaine. Le projet a été créé par la Coopérative Il Gaviale avec le soutien de la Province autonome de Trento et Hydro Dolomiti Enel, sous le patronage de la Ville de Dro, la région autonome du Trentin-Haut-Adige / Südtirol, Ministère du Patrimoine et de la Culture, Arcus - Art, culture, divertissement, Project Performing Arts Promotion APAP, Programme communautaire / Culture européenne. L'établissement propose, en plus des résidences pour de jeunes artistes, un soutien hautement qualifié pour les phases de travail allant de la conception à la vision, de la production à la coproduction pour de nouveaux projets artistiques et spectaculaires, nationaux et internationaux 35 .

La tendance à organiser des manifestations culturelles au sein des bâtiments est de plus en plus populaire. Ce sont les mêmes organisations qui détiennent la propriété des systèmes hydrauliques à promouvoir ce type d'activité. Depuis 2002, par exemple, l'Ente Nazionale per l'Energia Elettrica (Enel), favorise l'initiative Centrali aperte. Enel organise des journées de visite de ses centrales, afin de sensibiliser et de faire connaitre les procédures et les normes environnementales concernant la production d'électricité.

Parmi celles-ci, la centrale Taccani ${ }^{6} 6$ de Trezzo d'Adda (Milano) représente un cas célèbre. Conçue par Gaetano Moretti au début du XXe siècle, elle produisait l'énergie pour alimenter l'industrie cotonnière naissante de Cristoforo Benigno Crespi, dans le village de Crespi d'Adda. La centrale est encore en fonctionnement et en plus des visites guidées, des spectacles musicaux et de théâtre sont proposés, ainsi que des expositions d'art ou des collections d'art et objets d'époque.

\section{Les musées de l'histoire énergétique locale}

Dans de nombreux cas la fonction productive des centrales hydroélectriques devient l'objet d'activités éducatives dans le contexte d'un musée. Ce dernier vise à la diffusion des connaissances sur l'histoire de l'édifice dans ses composantes architecturales et technologiques, dans une optique qui s'étend au contexte historique local.

La centrale de Cedegolo est l'un des exemples les plus frappants du musée de l'hydroélectricité dans le nord de l'Italie. La centrale est située dans la province de Brescia, en Lombardie. Désaffectée depuis 1962, elle a été acquise par la Mairie de Cedegolo, qui l'a utilisée pour accueillir le musée de l'hydroélectricité37. L'exposition reconstitue les étapes clés de l'industrialisation italienne et les événements historiques de la vallée, profondément marquée par l'exploitation de sa richesse hydrique $^{38}$. Le musée fait partie du Musée de l'Industrie et du Travail de Brescia (Musil), un système qui outre le Musée de Cedegolo, comprend le Musil Rodengo Saiano, entrepôt ouvert au service de tout le système des musées et le Musée du Fer. Par contre, les projets répétés pour la construction d'un siège principal du Musil dans l'ancienne usine Tempini à Brescia n'ont pas encore été mis en œuvre39. 
La region Trentin-Haut-Adige sauvegarde le patrimoine industriel d'une façon remarquable. Ouvert en 1998, le Museo dell'energia elettrica, un petit musée de l'hydroélectricité se trouve à Serso dans la province de Trento. Sous le patronage de la municipalité de Pergine, il a été conçu par la Société multiservices de l'énergie et de l'environnement, Amea (maintenant Servizi Territoriali Est Trentino Società per Azioni).

Le musée est inséré dans une centrale encore en fonctionnement et elle est accessible au public, ce qui permet au visiteur d'une part de percevoir l'évolution technologique et, d'autre part, d'apprécier la clairvoyance et l'obstination de ceux qui ont conçu, déjà en 1893, la première usine hydroélectrique triphasée de l'Empire des Habsbourg.

Activée en 1893 sur la rivière Noire, dans la vallée de Serso, la centrale a favorisé l'augmentation de la productivité des ateliers locaux (moulins, scieries, usines) et elle a permis l'éclairage des maisons et des rues.

Aujourd'hui, la technologie a apporté des améliorations significatives au groupe turbine alternateur synchrone massif d'un temps, en le remplaçant par un autre plus petit et automatisé.

L'ancienne structure, un véritable joyau de la technique pionnière, n'a pas été démolie: l'importance de l'édifice a poussé la communauté de Pergine à suggérer l'idée d'un projet de musée, dans le but de recueillir les témoignages de l'histoire de la centrale ${ }^{40}$.

\section{Problématiques contemporaines pour la conservation du patrimoine hydroélectrique : les cas de Vérone, Vénétie}

En 2004, la Région de la Vénétie, les Soprintendenze régionales et le Fondo per l'Ambiente Italiano (FAI), ont organisé un colloque qui a permis de réfléchir sur la question de la protection de l'eau en tant que composante du paysage et, par conséquent, de l'identité de la région de la Vénétie. Les journées d'étude ont eu lieu à Bassano del Grappa. La conférence portait sur « $L a$ memoria dell'acqua. Conoscenza e valorizzazione dei sistemi idrici nel paesaggio veneto ».

Dans l'introduction aux actes41, le professeur Guido Zucconi a réitéré la nécessité d'une approche multidisciplinaire au patrimoine culturel, pour une « archéologie du paysage hydraulique » nécessaire à la compréhension systématique de l'ensemble ${ }^{42}$.

Pour faire de l'archéologie dans ce domaine, il est nécessaire d'étudier le phénomène hydroélectrique dans le domaine historique de l'architecture, de l'urbanisme, et aussi de l'ingénierie, de la géomorphologie, de la biologie, de l'aménagement paysager, de l'économie et de la société. Une approche qui a été adoptée en partie dans un projet d'étude territoriale, réalisée en 2008 sur le développement de l'hydroélectricité en Vénétie.

Le projet était soutenu par la Région Vénétie, dans le cadre du protocole d'accord entre la Région de la Vénétie, l'Université Ca 'Foscari de Venise et l'IUAV. Il portait pour la recherche sur le catalogage et la production de documents utiles à l'approfondissement de l'histoire vénitienne de la première décennie du $\mathrm{XX}^{\mathrm{e}}$ siècle, dans la période d'entre-deux guerres "Il Veneto tra le due guerre : $1918-1940 » 43$. Le projet en ligne analyse également certaines centrales hydroélectriques qui ont été bâties dans la région de Vérone entre les années trente et cinquante. L'étude se limitait à fournir leur emplacement et l'iconographie historique correspondante.

Les bâtiments dont on parle sont relativement récents, donc, il serait compréhensible de comprendre, pas de partager, les raisons pour lesquelles ils ne sont pas encore devenus partie du patrimoine culturel local. Il n'est pas acceptable, cependant, que même les premiers exemples de centrales hydroélectriques, bâties à Vérone à partir de la fin du XIXe siècle, n'appartiennent pas à la catégorie du patrimoine sauvegardé. 
Les raisons qui empêchent les témoignages historiques d'industrialisation qui se trouvent à Vérone d'être correctement sauvegardés et valorisés sont nombreuses. Il y a deux aspects principaux: le premier aspect concerne le rôle secondaire qui a été joué par l'industrie en faveur d'une plus forte vocation agricole, commerciale et militaire44. Le deuxième aspect concerne la richesse en monuments anciens et objets d'art du patrimoine culturel de la ville de Vérone, dont la protection est prioritaire, au détriment du patrimoine bâti des $\mathrm{XIX}^{\mathrm{e}}$ et XX $\mathrm{XX}^{\mathrm{e}}$ siècles.

Vérone vit de deux identités différentes: la première s'identifie à la ville ancienne, située à l'intérieur des murs et patrimoine UNESCO ; elle est fortement liée aux services et aux activités touristiques. L'autre identité est représentée par la ville en dehors des murs antiques et est considérée à tort périphérique. Cette partie de la ville, suite aux désaffectations industrielles, vit une situation de subordination et de manque d'appréciation par les citoyens eux-mêmes, qui reconnaissent l'unique ville dans le centre historique.

Les études sur les réseaux d'infrastructure pour la production d'énergie dans le territoire de Vérone ont mis l'accent sur le canal Camuzzoni45, le premier canal de la ville industrielle géré par le consortium Canale Industriale Camuzzoni. En effet, le réseau hydraulique est beaucoup plus complexe et il voit une distribution capillaire de centrales hydroélectriques. Une partie du système hydroélectrique local est géré par l'Azienda Generale dei Servizi Municipalizzati (AGSM), le reste par le Groupe Enel. Actuellement, les initiatives visant à la connaissance et à la protection de ce type de patrimoine sont à leurs débuts. Certaines de ces initiatives comprennent des activités promues par les associations de cyclotourisme. Des visites guidées ont été organisées, mais de façon sporadique.

La situation critique qui caractérise certains de ces objets historiques a mis en évidence la nécessité d'une réflexion, en vue de leur future mise en valeur.

Parmi les cas ${ }^{46}$, on a choisi celui de la centrale Tombetta I et II, du type au fil de l'eau, le long du canal Camuzzoni, bâtie dans les années vingt et agrandie dans les années 1940. Propriété de AGSM, la centrale a été gérée par le Consorzio Canale Industriale Camuzzoni47 jusqu'aux premiers mois de 2013. Des restaurations d'une ampleur considérable ont eu lieu depuis la Seconde Guerre mondiale jusqu'en 1991, tandis que le dernier remplacement de la machinerie a eu lieu entre 2003 et 2006, une période durant laquelle ont été installés sept turbines Kaplan de type «bulbe ", contenant un générateur immergé dans l'eau. La production de l'usine est de 60 millions de $\mathrm{KW} / \mathrm{h}$.

Depuis 2000, la centrale Tombetta I et II a été automatisée et un système informatique en ligne gère son fonctionnement. La centrale produit de l'énergie sans arrêt et le système informatique peut fonctionner et arrêter le mouvement des turbines au besoin. L'énergie produite est canalisée dans les centres de distribution dispersés dans la ville. Le personnel surveille les opérations à distance. Ce qui reste de la vieille machinerie se trouve aujourd'hui sur l'aire de trafic, en face de la centrale. Depuis les années 1950, la salle de contrôle est inutilisée et sera bientôt démantelée. Il n'y a pas de mesures de protection qui garantissent le respect de l'authenticité de ce monument historique.

À cet égard, même le dernier document concernant la protection du patrimoine industriel, les Principes de Dublin, ne donne que des lignes directrices encore trop générales et théoriques pour les sites ou ensembles industriels actifs $4^{8}$. Ce qu'on veut souligner, c'est qu'en dépit de la sensibilité et de la réglementation pour la protection du patrimoine industriel, lors de la récupération, les modalités d'intervention ne sont pas toujours claires: quels sont les choix à faire face à la nécessité de moderniser et de remplacer les machines et les systèmes de production?

Un deuxième cas concerne la centrale de Colombarolo, dans la province de Vérone. Il s'agit 
d'une structure au fil de l'eau, le long du canal Marazza. Le territoire est compris dans un méandre du fleuve Adige, qui correspond aujourd'hui au parc Adige Sud, un projet qui a été lancé dans les années 1990 et qui n'a pas encore été mis en œuvre par la municipalité. Le projet vise à développer un territoire abandonné d'une grande richesse. Les structures architecturales de valeur font de ce territoire un point d'intérêt de grande importance, même du point de vue du patrimoine industriel, grâce aux canaux, barrage et centrales hydroélectriques, mais surtout dans une optique plus élargie liée à la connaissance et à l'interprétation de la mémoire historique du territoire. La centrale fut conçue par l'ingénieur Ettore Marazza et construite au début du XX $\mathrm{XX}^{\mathrm{e}}$ siècle. L'énergie produite était utilisée pour alimenter le châssis de la Manufacture Festi Rasini de San Giovanni Lupatoto et pour éclairer les routes du noyau urbain49. Par la suite, lorsque la Société Electrique Milani (SEM) en a acquis la propriété, la centrale a été utilisée comme une réserve50, conduisant à la perte d'importance de l'usine, jusqu'à la récente élimination des machines. Aujourd'hui, le bâtiment est vide et il se trouve dans un très mauvais état de conservation. Le toit de la centrale est tombé et en raison du manque de productivité, la centrale ne revêt aucun intérêt économique. Sera-t-il possible de maintenir l'identité de ce système hydroélectrique dans le cas où la centrale serait démolie ?

Les études d'archives relatives aux événements historiques concernant la centrale a permis de comprendre que la réalisation des œuvres a été la cause de la rupture de l'équilibre qui a dominé pendant des siècles dans le territoire. Toutes les propriétés foncières ont été expropriées ${ }^{11}$, entraînant l'éloignement de la présence civile sur le territoire.

Dans le cadre d'une archéologie du paysage hydraulique, nous devons reconstruire la «stratification mémorielle »52. L'augmentation de la valeur du paysage contemporain de ces lieux doit être comprise en tenant compte de la présence d'eau et d'œuvres pour leur règlement, comme les couches les plus superficielles d'une sédimentation historique complexe. Cela devient important afin de connaître si les cultures préexistantes ont été décisives dans le choix de l'emplacement pour la construction de centrales hydroélectriques.

Dans les cas mentionnés dans le premier chapitre, on a vu comment les éléments technologiques sont essentiels afin de donner une identité au bâtiment. En vue de la mise en valeur du paysage hydroélectrique de cette zone, si le bâtiment est démoli, le canal qui était utilisé pour la production d'énergie, suffira-t-il à évoquer l'ancienne vocation productive?

Comme on l'a vu dans l'introduction de cet article, il est probable que, sous peu, de nouvelles turbines puissent être utilisées pour exploiter les canaux existants.

En vue de cette possible double destination du parc, on devrait établir des règles pour permettre la coexistence entre l'industrie et le loisir. La tendance à l'isolement ne sera plus acceptable ; la nouvelle activité industrielle devra être compatible au regard des priorités établies par le système du parc, selon les bonnes pratiques concernant la production d'énergie associée à la culture.

1 Pavia Rosario, «Sentieri elettrici», Pavia R. (dir.), Paesaggi
elettrici. Territori, architetture, culture, Venezia, Marsilio Editori, 1998, p. 339.

2 Pavia Rosario, «Sentieri elettrici», loc. cit., p. 339-347.

3 Zucconi Guido, «L'acqua nella sua dimensione urbana e territoriale», Spiazzi A.M., Zucconi G. (dir.), La memoria dell'acqua. Conoscenza e valorizzazione dei sistemi idrici nel paesaggio veneto, Padova, Unipress, 2004, p. 87-90.

$4 \mathrm{Au}$ niveau mondial, on fait référence au Centre International sur la Petite Hydraulique (CIPH), directement sous les auspices de l'Organisation des Nations Unies pour le Développement 
Industriel (ONUDI), le Ministère des Ressources de l'Eau et le Ministère du Commerce de la République populaire de Chine. Il est le quartier général du Réseau International sur la Petite Hydraulique (RIPH), une organisation internationale comptant plus de 400 membres dans 70 pays. L'objectif du CIPH est la promotion du développement mondial de la petite hydraulique. www.inshp.org

5 Par exemple, on se réfère à la liste des œuvres d'art pour la production hydroélectrique qui a été mis à jour par le Sistema Informativo Lombardo per la Valutazione di Impatto Ambientale (SILVIA), et qui concerne 187 projets, présentés entre 2006 et 2012 pour la réalisation de centrales ou pour la réhabilitation de systèmes existants dans la Région Lombarde. http://silvia.regione.lombardia.it/silvia/jsp/procedure/elencoPr ocedure.jsf;jsessionid=94EB617665ECE681269178EC964D7C62 .tomcat 5 ?idSel $=61 \& p K e y=$ OPERA01REGVER\&pKey $=$ OPERAo 1 REGVER\&etich=Impianti\%2oidroelettrici\&pProv=-1

6 Pour plusieurs information sur la diffusion du mini hydro en Italie, cf.: Giudici Giancarlo, Ghisla Fulvio, Sottocornola Riccardo, "The Potential for Mini-Hydro in Italy: The Case of the Refurbishment of Old Mills and Workshops », Politecnico di Milano, Contribution to the World SHP Development Report, 2011, p. 1-5.

7 Par rapport aux grandes centrales électriques, la technologie du mini hydro a un impact limité sur l'environnement, même s'il n'est pas encore complètement estimé. Il est capable de fonctionner avec une efficacité de conversion d'énergie élevée (sans nécessiter de gros travaux d'entretien). Le mini hydro peut représenter une solution dans le rétablissement de vieux bâtiments industriels désaffectés ou des centrales. Boano Fulvio, Camporeale Carlo, Cavagnero Paolo, Fenoglio Stefano, Ravelli Roberto; Ridolfi Luca, Mini Hydro e impatti ambientali. Sintesi organizzata dello stato dell'arte scientifico, Politecnico di Torino,Torino, 2011, p. 33-34.

8 On souligne qu'avec l'utilisation du terme « paysage » on fait référence à l'ensemble des perceptions humaines liées à la vision et la connaissance des éléments naturels et humains d'un lieu particulier.

9 Eugenio Turri (1927-2005) fut un des auteurs qui a écrit sur la question du paysage. Il affirme que la découverte du paysage est l'effet immédiat des dévastations portées par l'industrie. Turri Eugenio, Il paesaggio come teatro, Venezia, Marsilio, 2003, p. 110. Et aussi: Fontana Vincenzo, Profilo di architettura italiana del Novecento, Venezia, Marsilio, 1999, p. 85-95.
10 Le dernier essai de Salvatore Settis décrit d'une façon très lucide la «nuova desolante forma del paesaggio italiano». Settis Salvatore, Paesaggio costituzione cemento, Torino, Einaudi, 2010, passim.

11 En faisant référence à André Guillerme, cf. Zucconi Guido, «L'acqua nella sua dimensione ...», loc. cit. , p. 88.

12 Pavia Rosario, «Sentieri elettrici», loc. cit., p. 339.

13 Les valeurs de l'industrie diffèrent selon l'époque historique, la sensibilité, la culture et le point de vue de l'observateur. Sur le thème, cf. Dansero Egidio, Vanolo Alberto, «Per una geografia del paesaggio industriale in Italia. Un'introduzione», Dansero E., Vanolo A. (dir.), Geografie dei paesaggi industriali in Italia, Milano, Franco Angeli, 2006, p. 11-16.

14 Monti Guglielmo, «Domani l'acqua», loc. cit., p. 207.

15 http://www.centrovolta.it/laviadellenergia/inglese/index.htm

16 Association à but non lucratif, fondée à Milan en 2001, qui promeut une approche critique dans le contexte des biens et des droits culturels. Ar.Me.S. est en charge de la mise en œuvre de méthodologies et de projets proposant une attention particulière à la dynamique du territoire et à ses façons de le gouverner. L'association organise des conférences, des séminaires, des formations, des expositions sur la recherche liée aux arts, de la musique, des médias, de la culture et des musées d'entreprise. 17http://www.centrovolta.it/laviadellenergia/inglese/territorio/t rezzo.htm

18 Pour plus d'information concernant la constitution du parc: http://www.parcoaddanord.it/

19 Pour un approfondissement sur le parc et ses monuments historiques, cf. " Paderno d'Adda, storie di acqua e di uomini», Paderno d'Adda (CO), Habitat, 1989.

20 Manfredini Achille, «L'impianto idro-elettrico alle rapide dell'Adda presso Paderno", Il Monitore Tecnico, III, 20 ottobre 1897, III, p. 161-169. Bogo Antonio, «Centrale idroelettrica Bertini», Garlandini A., Negri M. (dir.), I monumenti storicoindustriali della Lombardia. Censimento regionale, Milano, Regione Lombardia, 1977, p. 480-481.

21 Bogo Antonio, «Centrale idroelettrica Esterle», Garlandini A., Negri M. (dir.), I monumenti storico-industriali, loc.cit. p.482483 . 
22 Bogo Antonio, «Centrale idroelettrica Semenza», Garlandini A., Negri M. (dir.), I monumenti storico-industriali, loc.cit. p., 456-457.

23 En 2012, le cas du pont de Paderno d'Adda a été l'objet d'étude dans la cadre du projet tutoré «Euvres du génie», 5ème promotion du Master TPTI (Techniques Patrimoine et Territoires de l'Industrie); chef du projet : Professeur Ana Cardoso de Matos, tuteurs: Benjamin Ravier, Erika Bossum, João Santos; étudiants : Wendy Espinal, Mariangela Licordari, Daniel Maldonado. Site web http://ouvresdegenie.wordpress.com/

24 CRESPI CULTURA - Associazione Culturale Villaggio Crespi encourage la connaissance de l'histoire du patrimoine industriel de la Company Town du village ouvrier de Crespi d'Adda. http://www.villaggiocrespi.it/en/ et bibliographie essentielle : http://www.villaggiocrespi.it/crespidadda/testi/

$25 \mathrm{http}: / /$ www.museialtovicentino.it/en/

26 Pavia Rosario, «Sentieri elettrici», loc. cit., p. 344.

27 Les eaux des chutes des Marmore sont exploitées par la centrale de Galleto, donc le flux est libéré pour les touristes à certains moments pendant les différentes saisons de l'année:

http://www.marmorefalls.it/home.php?lang=eng

Sur l'exploitation des eaux dans le territoire de Terni, cf. Covino Renato, Gallo Giampaolo, Le industrie di Terni. Schede su aziende, infrastrutture e servizi, Città di Castello (Pg), Crace, 2004.

28 http://www.tiefenbrunner.com/en/estate/hydroelectricpower-station.html

29http://www.comunefortezza.eu/system/web/zusatzseite.aspx ?menuonr $=218613599 \&$ detailonr $=218255622 \&$ sprache $=3$

\section{0 http://www.tecneum.eu/index.php}

31 La Fondation s'occupe des biens matériels et immatériels du patrimoine industriel de la société A2A : http://www.fondazioneaem.it/

32 Née en 2008, A2A est le résultat de la fusion sociétaire des anciennes sociétés municipales de Milan (AEM et AMSA) et Brescia (ASM).

33 Longo Angelo, Visintainer Claudio, Acquaenergia. Storia e catalogazione delle centrali idroelettriche del Trentino, Trento, esaExpo 2008.
34 Sur les contenus de l'exposition, cf. MENINI Giacomo, $I$ luoghi dell'acqua. Architetture e paesaggi delle centrali elettriche in Valtellina, Verona, Grafiche Aurora, 2013.

\section{5 www.centralefies.it}

36 Selvafolta Ornella, «La costruzione del paesaggio idroelettrico nelle regioni settentrionale», Pavia R., Paesaggi elettrici. Territori, architetture, culture, Venezia, Marsilio, 1998, p. 46-48.

37 http://www.musilbrescia.it/english/sedi/cedegolo/

38 Simoni Carlo, «Musei e percorsi del lavoro nel Bresciano », Fontana G.L. (dir.), Archeologia industriale in Italia. Temi, progetti, esperienze, Quaderni di patrimonio industriale, 1, Brescia, Aipai Grafo, 2005, p. 157-158; Acqua , natura e tecnica: il Museo dell'energia Idroelettrica di Cedegolo, Azzoni G. Simoni C., Brescia, Centro Servizi Musei, 2009.

\section{9 http://www.musilbrescia.it/english/ilmusil/default.asp}

40 « Guida al Museo della centrale idroelettrica di Serso», Anderle Renzo, et alii, Pergine, Publistampa arti grafiche, 1999.

41 La memoria dell'acqua. Conoscenza e valorizzazione dei sistemi idrici nel paesaggio veneto, Spiazzi A.M., Zucconi G. (dir.), Padova, Unipress, 2004.

42 Zucconi Guido, «L'acqua nella sua dimensione ...», loc. cit. , p. 90 .

43 http://circe.iuav.it/Venetotra2guerre/

44 Pour une analyse de la situation urbanistique contemporaine de la ville de Vérone, cf. MASSIGNAN Giorgio, La gestione del territorio e dell'ambiente a Verona Repetita non iuvant. Verona, Scripta Edizioni, 2013.

45 Les essais concernant les événements qui ont porté à la réalisation du canal Camuzzoni sont nombreux, parmi eux, on fait réfèrence à: Caló Antonio, «Il canale Camuzzoni», Mancuso F. (dir.), Archeologia industriale nel Veneto, Venezia, Silvana Editoriale, 1990, p. 147-148; Caló Antonio, "Cronistoria di un progetto per l'industrializzazione di Verona: il canale Camuzzoni», Zangarini M. (dir.), Il canale Camuzzoni. Industria e società a Verona dall'Unità al Novecento, Verona, Cierre, 1991, p. 151-193. Petri Rolf, «Tra municipio e ciminiera. Pubblico e privato nella prima età industriale», Zangarini M. (dir.), Il 
canale Camuzzoni...» op.cit., p. 137-150. Morgante Michela, Il canale e la città. Il Consorzio canale Camuzzoni nel primo Novecento, Verona, Cierre 2006.

46 L'étude fait partie du projet de doctorat "Il sistema integrato ' irriguo elettrico' veronese di derivazione atesina: vicende storiche e prospettive di valorizzazione socio culturale'". Dottorato in Beni Culturali e Territorio, Scuola di Dottorato in Studi Umanistici, Università degli Studi di Verona, XVI ciclo, 2011-2013; doctorante: Erika Bossum; tuteur: prof.ssa Daniela Zumiani.

47 Le consortium Canale Industriale Camuzzoni possède des archives très riches et bien conservées qui concerne les œuvres d'art dont la propriété est municipale. Pour les autres cas, comme pour la plupart des cas italiens qui ont été acquis par Enel, les archives se trouvent entièrement aux archives historiques de la Société, à Naples. http://enelikon.enel.it

48 Précisément, l'article 8 des Principes de Dublin, «Principes Conjoints ICOMOS-TICCIH pour la conservation des sites, constructions, aires paysages et du patrimoine industriel», adoptées par la 17e Assemblée générale de l'ICOMOS le 28 novembre 2011.

49 Archivio Storico San Giovanni Lupatoto, Ufficio Tecnico del Comune di San Giovanni Lupatoto, b. 132.

50 Archivio Storico Enel, Ex Compartimento di Venezia, Società Elettrica Milani, b. Festi Rasini.

51 Archivio Storico Enel, Ex Compartimento di Venezia, b. Proprietà Pestrino; b. Nuovo canale Colombarolo Bassona.

52 Daumas Jean-Claude, La mémoire de l'industrie. De l'usine au patrimoine, Paris, Presses Universitaires de France-Comté, 2006, p. 15 . 